\title{
Rapid Detection of Candida species Isolated from Denture Stomatitis Patients using Phenotypic methods and Chromogenic agar media
}

Haifa Abdul Ghani Abdulla BSc., Master Student

Eman A. Mustafa BSc, MSc.(Asst.Prof.)

\author{
Department of Dental Basic Sciences \\ College of Dentistry, University of Mosul \\ Department of Dental Basic Sciences \\ College of Dentistry, University of Mosul
}

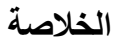

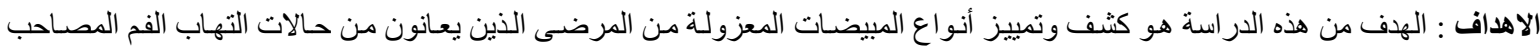

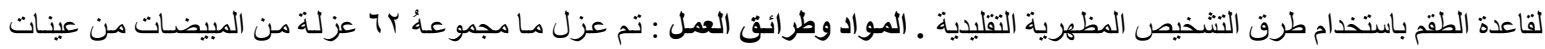

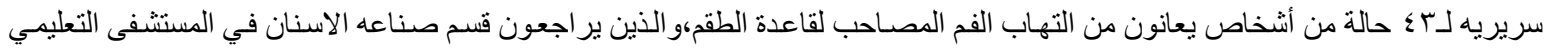

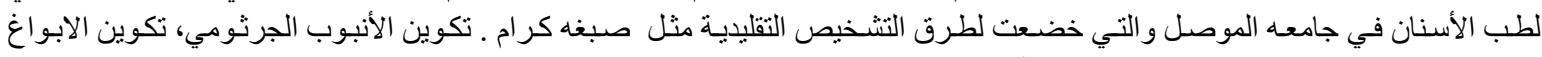

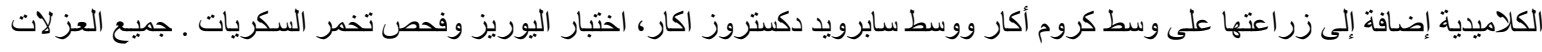

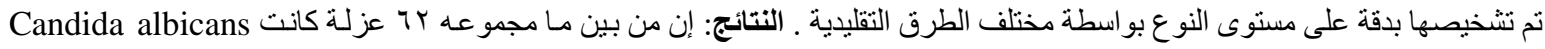

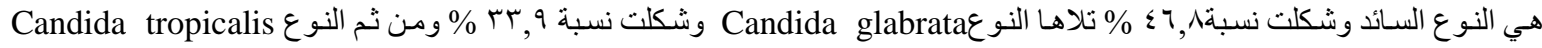

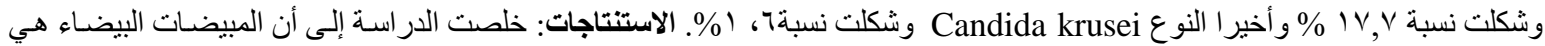

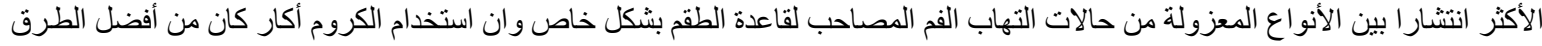

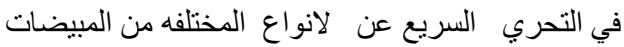

\begin{abstract}
Aims: The purpose of this study was Isolation and phenotypic identification of different candida species isolated from denture stomatitis patients. Materials and Methods : Over all 62 isolates of Candida species yielded from 43 patients attending prosthodontic department|College of DentistrylMosul universitylDental teaching Hospital. Clinically, the samples processed by traditional methods including culture characteristics, gram staining reaction, germ tube, chlamydospore formation, culture on CHROM agar Candida , Sabouraud Dextrose agar, urea's test and Carbohydrates fermentation test. Results: All isolates were accurately diagnosed to the species level by various traditional methods, among the total of 62 Candida species, the predominant type was the Candida albicans which accounted for $(46.8 \%)$ followed by Candida glabrata (33.9\%), Candida tropicalis (17.7\%), finally the species Candida krusei accounted for (1.6\%). Conclusions: Candida albicans is highly spreading among patients wearing dentures particularly those suffering from denture stomatitis in which CHROM agar Candida medium is best method for rapid identification and documentation of different Candida species .
\end{abstract}

Key words: Candida species, Denture stomatitis, Phenotypic methods, CHROM agar Candida medium.

Abdulla HA., Mustafa EA. Rapid Detection of Candida species Isolated from Denture Stomatitis Patients using Phenotypic methods and Chromogenic agar media. Al-Rafidain Dent J. 2020 ;20 (1):125-133.

DOI: $10.33899 /$ rden.2020.126821.1029

Received: 19/3/2020 Sent to Referees: 20/3/2020 Accepted for Publication: 26/4/2020




\section{INTRODUCTION}

Candida species are regarded the most common isolated and the frequent etiological factor of denture stomatitis ${ }^{(1)}$ Approximately 300 Candida species are documented ,10\% can cause infections in human ${ }^{(2)}$. Different species of Candida are described as a communal microorganisms, they exist in 30-60\% of healthy people and $60-100 \%$ of patient with denture stomatitis ${ }^{(3,4)}$, non albicans species including Candida glabrata, Candida tropicalis, Candida parapsilosis, and Candida krusei etc .also appeared as etiological agents in oral candidiosis either as a co-infection with C.albicans or as a sole pathogenic microorganism $^{(4,5)}$. there are different conventional methods for identification of yeast such as: culture on sabouraud dextrose agar (SDA) media ,corn meal agar ( CMA), germ tube test, carbohydrate fermentation test , and in order to accelerate detection of candida CHROM agar are used as a selective and differential media which inhibit the growth of any microorganisms except candida ${ }^{(6)}$. The component of this media ,yeast extract,chromopepton ,chromogen mixture ,chloramphenicol and agar allowing identification of candida isolates by their color and colony aspect, special colony with different colors appear on this media due to chromogenic substrates which react with enzymes produced by candida therefore , chrmogenic media has the ability to differentiate mixed candida and yeast infections (6)

\section{MATERIALS AND METHODS}

\section{1- Sample collection :}

patient selection in this study including: elderly adult patients ,suffering from stomatitis . Their ages range (50-85)years old, wearing complete denture, the age of denture range (5-20) years, patients who have taken antifungal drugs were excluded from the samples.,at least three month ago . Swaps were gained from 43 patients who attending Dental teaching Hospital I College of Dentistry \Mosul university. Specimens were collected after clinical diagnosis by scraping sterile swabs across the palatal mucosa in contact with the denture and the inner surface of denture , then swabs were transferred into $2 \mathrm{ml}$ sterile nutrient broth vials.

\section{Microbiological work}

All samples were primarily cultured on sabouraud dextrose agar(SDA) adding chloramphenicol $50 \mathrm{mg} / \mathrm{L}$ to inhibit bacterial growth, and incubated at $37 \mathrm{C}^{0}$ for 24-48 hrs, to ensure that all Candida spp in samples were isolated, The identification to species level was carried out :

A- Gram staining reaction for all specimens .

B- Germ tube production (GT) .

To differentiate between $C$. albicans from non albicans groups, human blood was 
placed in test tube and left in rack to clot, after clotting the sample centrifuged at 3000 rpm for $10 \mathrm{~min}$, serum was separated carefully and placed in sterile test tube and then colony was picked up with sterile loop and was placed in $0.5 \mathrm{ml}$ of serum, this mixture was placed in an incubator at $37 \mathrm{C}^{0}$ for 3-4 hrs. Adrop of this mixture was placed, put on a clean slid, overlaid with a clean cover slip and examined by high power objective lens of microscope $(40 x)^{(7)}$

C- Chlamydospor production :

The colony was picked up from selective media and then was cultured on $1 \mathrm{~cm} \times$ shape in corn meal agar, covered with sterile cover slip the plate incubated at room temperature for $48-72$ hrs. then examined under $40 \mathrm{x}^{(8)}$.

D- Chromogenic agar medium :

All samples were cultured on to CHROM agar Candida ( Hi media, M.I.D.C., India ) ,then the plates were incubated at $37 \mathrm{C}^{0}$ for 24-48 hrs. ${ }^{(9,10)}$
E- Carbohydrate fermentation test :

This test based on sugars fermentation and prepared according to Knenman et al. ${ }^{(11)}$

F- Ureas's test : The isolated colonies was inoculated on urea base agar within test tubes and after incubation the color of indicator (phenol red ) was changed from yellow to pink color as a positive result $(9,12)$.

The results were analyzed statistically by using Q square ,all data analyzed statistically significant value at $p<0.05$ was considered by using sigma sat program. ${ }^{(13)}$

\section{RESULT}

The distribution of Candida species in this study exhibited no significance in both sex male and female $(p=0.388)$, Figure (1).

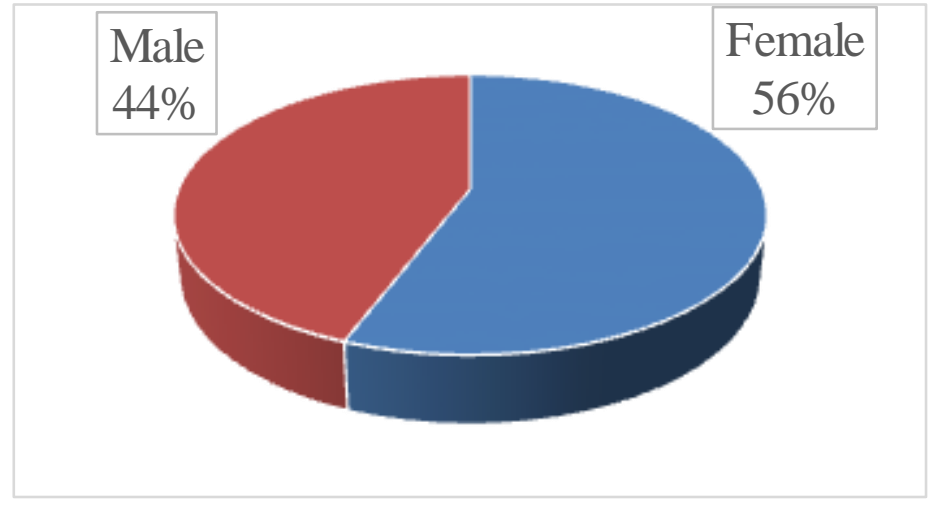

Figure (1): distribution of Denture Stomatitis in male and female 
This study was achieved to identify different species of candida in clinical samples. Twenty nine isolates of C.albicans identified by germ tube test, while other Candida species were negative. Twenty nine species of C.albicans produce chlamydospore and only two species of C.tropicalis gave positive result (Table1).

Table (1) : Identification of Candida species by germ tube and chlamydospore formation

\begin{tabular}{|c|c|c|c|c|c|}
\hline \multirow[t]{2}{*}{ Candida species } & \multirow[t]{2}{*}{ Total } & \multicolumn{2}{|c|}{ Germ tube formation } & \multicolumn{2}{|c|}{ Chlamydosopres formation } \\
\hline & & positive & Negative & Positive & Negative \\
\hline Candida albicans & 29 & 29 & 0 & 29 & 0 \\
\hline Candida glabrata & 21 & 0 & 21 & 0 & 21 \\
\hline Candida tropicalis & 11 & 0 & 11 & 2 & 9 \\
\hline Candida krusei & 1 & 0 & 1 & 0 & 1 \\
\hline
\end{tabular}

Out of 62 isolated Candida , 29(46.8\%) isolates were detected as $C$. albicans by CHRom agar, 21(33.9\%) isolates as C.glabrata, 11(17.7\%) isolates as C.tropicalis and only $1(1,6 \%)$ isolate of C.krusei (Table 2) the distribution of Candida species were shown in Figure (2) . gram's staining findings , the shape and the size of blastoconidia have been shown in (Figure 3). Germ tube and were chlamydospore formation tests were used for identification of Candida spp. (Figure 4) .

Table (2) : Identification of candida species by using CHRom agar

\begin{tabular}{|c|c|c|c|}
\hline Candida species & $\begin{array}{l}\text { Colony characteristic on } \\
\text { CHRomogenic agar }\end{array}$ & $\begin{array}{c}\text { No. of } \\
\text { isolates } \\
\text { total }(62)\end{array}$ & Percentage \\
\hline Candida albicans & Light smooth green colonies & 29 & $46.8 \%$ \\
\hline Candida glabrata & $\begin{array}{l}\text { White large glossy pale pink } \\
\text { colonies }\end{array}$ & 21 & $33.9 \%$ \\
\hline Candida tropicalis & Steel blue with violet shade & 11 & $17.7 \%$ \\
\hline Candida krusei & $\begin{array}{l}\text { Pink with white borders rough } \\
\text { colonies }\end{array}$ & 1 & $1.6 \%$ \\
\hline
\end{tabular}




\section{Denture Stomatitis Patients}

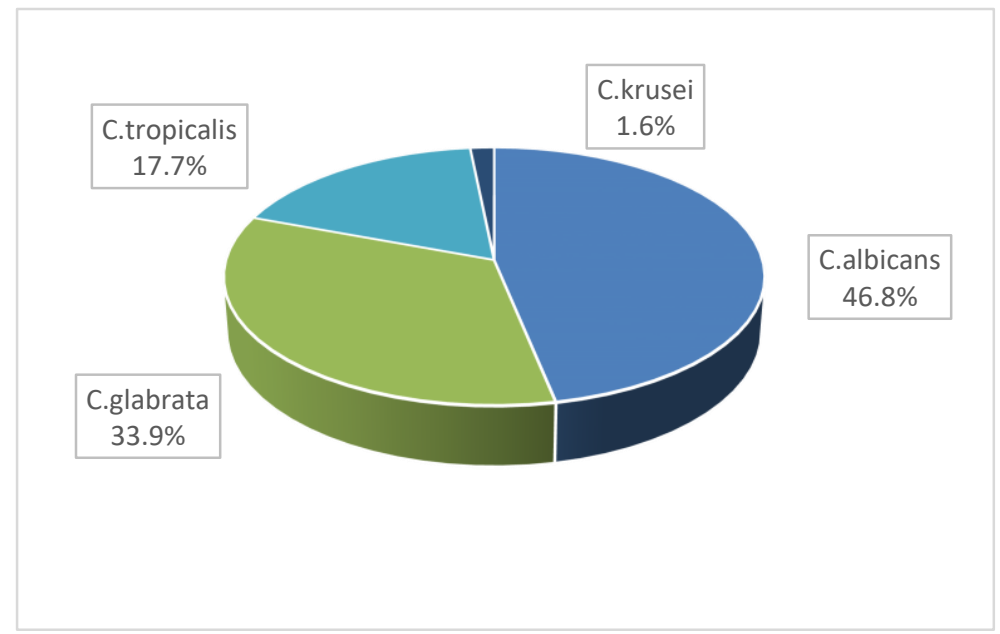

Figure (2): Distribution of Candida spp

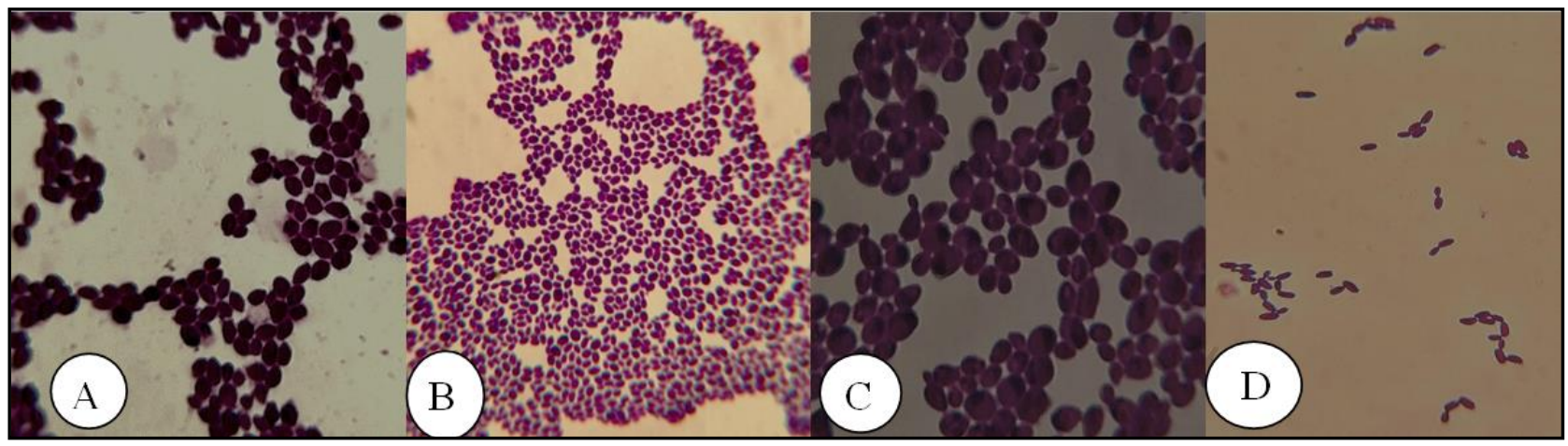

Figure (3): Gram stain of Candida species A- C.albicans B- C.glabrata C- C.tropicalis D- C.krusei

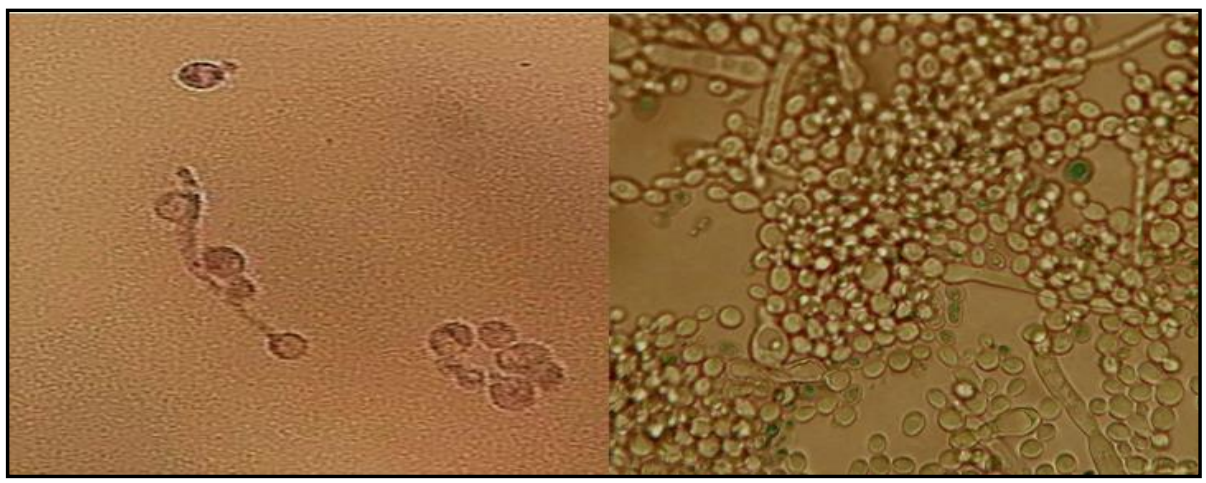

Figure (4): Detection of Candida spp. By Germ tube(left) and Chlamydospore formation (right) 
Different species of Candida were detected were used to confirm the growth of candida by culturing on SDA and CHROM agar isolates were shown in (Table 3) and only Candida medium (Figure 5). On CHROM agar, Candida krusei changed the urea base agar to the diverse species of Candida revealed pink color as a positive result of ureas test different color scolonies (Table 2). (Figure 6)

Carbohydrate fermentation and ureas test that

Table (3): Biochemical reaction of isolated Candida species

\begin{tabular}{cccccccc}
\hline \multirow{2}{*}{ Organisms } & \multicolumn{8}{c}{ Fermentation of charbohydrat } & \multirow{2}{*}{ Ureas's test } \\
& Glu & Mal & Suc & Lac & Gal & Treh & \\
\hline Candida albicans & $\mathrm{F}^{*}$ & $\mathrm{~F}$ & - & - & $\mathrm{F}$ & $\mathrm{F}$ & - \\
Candida glabrata & - & - & - & - & - & $\mathrm{F}$ & - \\
Candida tropicalis & $\mathrm{F}$ & $\mathrm{F}$ & $\mathrm{F} / \mathrm{V}^{*}$ & - & $\mathrm{F}$ & $\mathrm{F}$ & - \\
Candida krusei & $\mathrm{F}$ & - & - & - & - & - & + \\
\hline $\mathrm{F}^{*}=$ Fermenter & $\mathrm{V}^{*}=$ variable & & & &
\end{tabular}

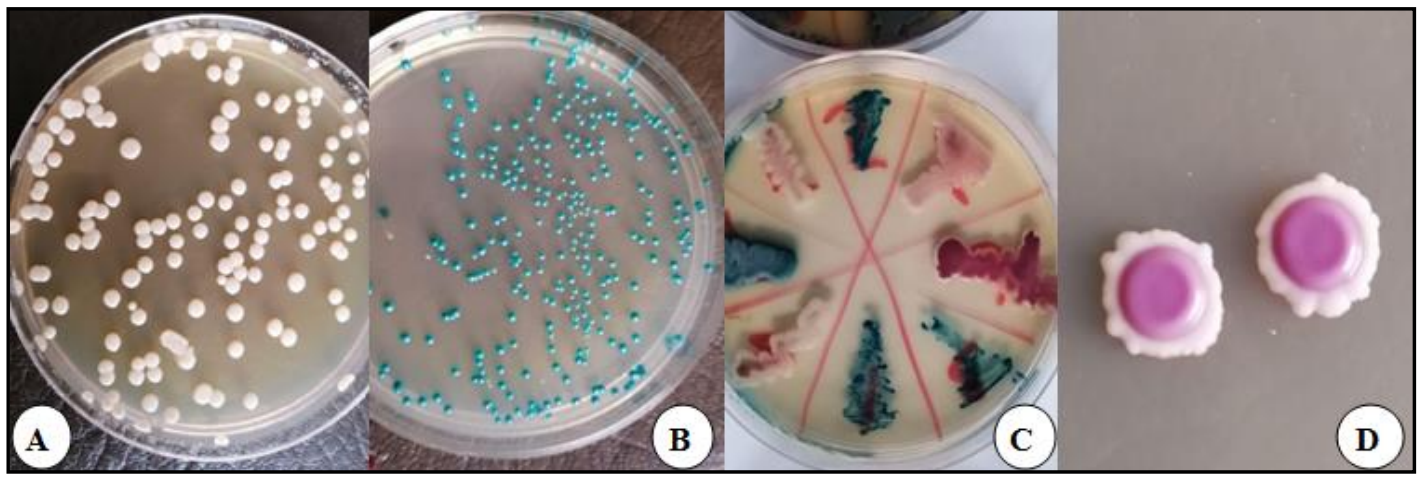

Figure (5) : (A) The colony of Candida spp. on SDA agar, (B) Candida albicans, (C)all Candida species,( D)Candida krusei on CHRom agar 


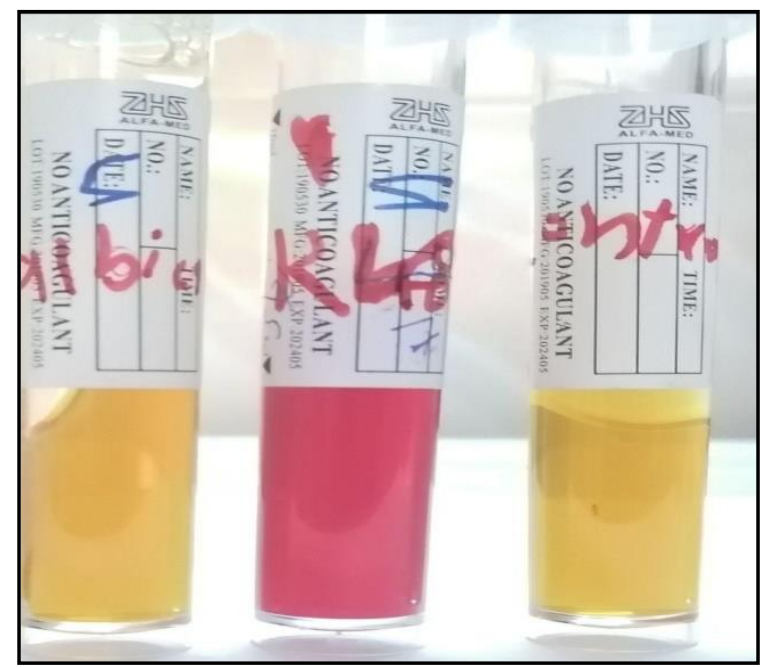

Figure (6) : Candida species on urea base agar

\section{DISCUSSION}

In present study C.albicans appeared as a predominant isolates in patients with DS agreed with other studies that found the same result $(14,15,16)$. The results of this study exhibited that there is no significant distribution of candida between male and female. all $C$. albicans strains and few strains of C.tropicalis form chlamydospore on nutritionally insufficient media such as corn meal agar. Thus this method can be used to determine these species and this result agreed with other studies, ${ }^{(2,9)}$ and all isolates of C.albicans form germ tube while the other species cannot produce it ${ }^{(9)}$ .depending on several studies carried out in universities and hospitals, $65 \%$ of denture users suffer from problems caused by candida albicans ${ }^{(17)}$

CHROM agar Candida medium is present as a novel medium which helped in isolation and differentiation of different species of
Candida according on different colors and morphology of colonies ${ }^{(4,9,18)}$. CHROM agar Candida medium in each liter contained peptone (10 g), glucose (20 g), agar (15 g), and chloramphenicol (0.5 g) and Chromogenic mixture. $(2 \mathrm{~g})$, while $\mathrm{pH}$ of the medium was maintained at 6.1according to instructions of manufacturer, for this reason this medium aided in differentiation between different species of Candida and this data was confirmed by another study carried out by Horvath et al ${ }^{(18)}$. in this study all 62 isolates which obtained from 43 patients produced distinct colors on CHROM agar and were grown well on this medium and this agrees with the truth that this medium act as a good differential medium, having good performance, less time consuming , having sensitivity for isolation and determination of Candida species ${ }^{(19)}$. Other study conducted by Lynn et al. noted that CHROM agar quickly identify C. albicans 
,C.glabrata, C.tropicalis and C. krusei based on different colors and morphological features of colonies CHROM agar candida gave a fast recognition repeatedly found yeast species which would usually be missed during traditional plating on solid media ${ }^{18)}$. Among 43samples grown on CHROM agar Candida medium plates ,twenty nine isolates of C.albicans showed Light smooth green colonies, twenty one isolates of C.glabrata showed White large glossy pale pink colonies, eleven isolates of C.tropicalis appeared as Steel blue with violet shade or as a dark blue colonies and one isolate of $C$. kruzei showed as pink with white borders rough colonies. (Table2) This characteristic of candida colonies on CHROM agar agreed with different study $10,20)$

\section{CONCLUSIONS}

In conclusion, this study reveals that CHROM agar Candida media having good potential for rapid identification of Candida species and this media can be used as a useful adjunctive media in the clinical laboratory for detection of Candida species.

\section{ACKNOWLEDGMENTS}

The authors thank the Department of prosthodontic and the Department of basic science in the college of Dentistry ,University of Mosul for supporting this study .

\section{REFERENCES}

1- Petrovic S, Barac M, Pficer J, Radunovic M, Jotic A, and Pucar A .Presence of different Candida species at denture wearers with type 2 diabetes and clinically healthy oral mucosa -pilot study . Balk J Dent Med. 2018; 22: 16-21.

2- Padmapriya G, Amshavathani S, and Percy Q. Molecular conformation of Candida species using self desined primers by PCR .Int J Curr Microbiol App Sc. 2015; 4(5):289-294.

3- Chauch L ,Pedrosa S, Gomes F, Esteves R, and da-Silva $\mathrm{S}$.Isolation of candida spp.from denture-related stomatitis . Brazilian J Microbiol. 2018; 49:148-151

4- Hussein Kh. Use of universal 18SrDNA gene and CHROMagar candida medium for the identification of Candida species isolated from denture wearers. Al-Kufa Univers J. Biol . 2016; special issue for $2^{\text {nd }}$ international for boil sci

5- Salerno C, Pascale M, Contaldo $M$, Esposito V, Busciolano M, Mililo L, Guida A, Petruzzi M , and Serpico R .Candida associated denture stomatitis. Med Oral Patol Oral Cir Bucal .2011; 16 (1): 139143.

6- Nigar I, Tarafdar S, khan R, Ahmad S and Abu Saleh A . Evaluation of Chromogenic agar media for rapid identification of candida species. Bangladesh $J$ Med Microbiol .2014; 9(1): 22-26

7- Gupta V, Abhisheik K, Balasundari S , Devandra N, Shadab K and Anupama M . Identifecation of Candida albicans using different culture media and its association 
in leukoplakia and oraL squamous cell carcinoma . JOMFP. 2019; 23(1): 28-35.

8- Bharathi R .Comparison of chromogenic media with the corn meal agar for speciation of Candida. JPURE APPL Microbiol .2018; 12(3) 1617-1622

9- Deorukhkar S and Roushani S . Identification of Candida speceis :Convential methods in the Era of molecular diagnosis .Ann Microbiol Immunol . 2018; 1 (1) : 1-10

10- Al-Ali I and Mejbel F. Isolation and identification of Candida spp from oral immune compromised patients with study of virulence factors 2014; Al-kufa University Biol .6(2): 115-123

11- Knenman E, Allen S, Janda W, Schreckenberger $\mathrm{P}$, and Winn $\mathrm{W}$, in color Atlas and Textbook of diagnostic microbiology $.4^{\text {th }}$ edn . Lippincott Philadelphia $1992 ; 791-878$

12- Al-jubouri M and Hassan A .Isolation of Candida spp. from patients with different types of leukemia who suffered oral candidiasis due to their weakened immune system. J Pharma Chem Biologic Scie. 2015; 3(1):79 -83

13- Wayne W., Daniel D. and Chad L.(2014).Biostatistics :Basic Concepts and Methodology for the Health Sciences .10 ed. Amazon Printer .USA.2014.

14- Peric M , Zivkovic R , Lemic A, Radunovic M, Milicic B and Arsenijevic A. The severity of denture stomatitis as related to risk factors and different Candida spp . Oral Surg. 2018; (Accepted Manuscript)

15- Altarawneh S, Bencharit S, Curran L, Mendoza A, Barrow D, Barros S, Preisser J, Loewy Zvi G, Gendreau L and Offenbacher S. Clinical and Histological Findings of Denture Stomatitis as Related to Intraoral Colonization Patterns of Candida albicans, Salivary Flow, and Dry Mouth. J. Prosthodont. 2013; 22:13-22.

16- Lazarin AA, Zamperini CA, Vergani CE, Wady AF, Giampaolo ET and Machado AL. Candida albicans adherence to an acrylic resin modified by experimental photopolymerised coatings: an in vitro study. Gerodontol. 2014; 31: 25-33.

17- Ahmad ZM, Mustafa EA, Jawad IA ,Adherence of Candida albicans to Flexible Denture Base Material .Al-Rafidain Dent J. 2012;12(2):229-235.

18- Malik U, Khan A, and Satti M , Comparative Evaluation of CHRom agar and API 20 CAUX in isolation and identification of candida species . JIIMC 2018; 13(2): 85-90

19- Abbas Z , Aziz S , Abdul-Mueed A . Epidemiological and Molecular Study for Candida spp in Vagina . Med. J. Babylon . 2013 ; 11(1) :110-119.

20- Golia S , Reddy M , Karjigi K and Hittinahalli V . Speciation of Candida using chromogenic and cornmeal agar with determination of fluconazol sensitivity . $A L$ Ameen J Med Sci . 2013 ; 6(2):163-166 . 